\title{
STABILIZATION OF BETTI TABLES
}

\author{
GWYNETH WHIELDON
}

\begin{abstract}
Let $I \subseteq R=\mathbf{k}\left[x_{1}, \ldots, x_{n}\right]$ be a homogeneous ideal generated by forms of degree $r$. We show here that the shapes of the Betti tables of the ideals $I^{d}$ stabilize, in the sense that there exists some $D$ such that for all $d \geq D, \beta_{i, j+r d}\left(I^{d}\right) \neq 0 \Leftrightarrow \beta_{i, j+r D}\left(I^{D}\right) \neq 0$. We also produce upper bounds for the stabilization index $\operatorname{Stab}(I)$. This strengthens the result of Cutkosky, Herzog and Trung that the Castelnuovo-Mumford regularity $\operatorname{reg}\left(I^{d}\right)$ is eventually a linear function in $d$.
\end{abstract}

\section{Background and results.}

1.1. Asymptotics of regularity of $I^{d}$. Let $\mathbf{k}$ be a field. For an ideal $I \subseteq R=\mathbf{k}\left[x_{1}, \ldots, x_{n}\right]$, much work has been done on showing that the Castelnuovo-Mumford regularity of $I^{d}$ is a linear function in terms of $d$ for high powers. The following theorem is a result of Cutkosky, Herzog and Trung:

Theorem 1.1 ([3, Theorem 1.1]). Let I be an arbitrary homogeneous ideal. Let $r(I)$ denote the maximum degree of the homogeneous generators of $I$. The following hold:

(i) There is a number e such that $\mathrm{reg}\left(I^{d}\right) \leq d \cdot r(I)+e$ for all $d \geq 1$.

(ii) $\operatorname{reg}\left(I^{d}\right)$ is a linear function for all d large enough.

They provide a criterion for estimating this $e$ in the case of an equigenerated ideal $I$, i.e., an ideal generated by homogeneous generators of the same degree. This result generalizes an earlier bound by Swanson

2010 AMS Mathematics subject classification. Primary 13A02,13D02, 13 C99.

Keywords and phrases. Free resolutions, Betti tables, stabilization, powers of ideals, regularity.

Received by the editors on June 13, 2011, and in revised form on September 19, 2011. 
giving the existence of $k$ such that

$$
\operatorname{reg}\left(I^{d}\right) \leq k d
$$

for homogeneous ideals in $[\mathbf{8}]$.

Let $I \subseteq R=\mathbf{k}\left[x_{1}, \ldots, x_{n}\right]$ be an ideal. The graded Betti numbers of a homogeneous ideal $I$ are given by $\beta_{i, j}(I)=\operatorname{dim}_{\mathbf{k}} \operatorname{Tor}_{i}(\mathbf{k}, I)_{j}$. The graded Betti numbers also correspond to the ranks of the free modules in a minimal free resolution of $I$. We organize this data into the Betti table of $I$ (in the style of Macaulay 2) displaying $\beta_{i, i+j}(R / I)$ in the $i$ th column and $j$ th row, as seen in Example 1.3.

Using techniques similar to those in $[\mathbf{1}, \mathbf{3}, \mathbf{6}]$, we produce here a sharper result on the asymptotics of Betti tables of powers $I^{d}$.

Theorem 1.2 (Theorem 4.1). Let $I=\left(f_{0}, f_{1}, \ldots, f_{k}\right) \subseteq \mathbf{k}\left[x_{1}, \ldots, x_{n}\right]$ $=R$ be an equigenerated ideal generated in degree $r$. Then there exists a $D$ such that, for all $d>D$, we have

$$
\beta_{i, j+r d}\left(I^{d}\right) \neq 0 \Longleftrightarrow \beta_{i, j+r D}\left(I^{D}\right) \neq 0 .
$$

This gives us that the shape of the Betti tables of powers of an ideal $I$ is eventually fixed, translated down by degree $r$ of the ideal.

Example 1.3. Let $I=\left(x_{3} x_{4} x_{5}, x_{1} x_{6} x_{7}, x_{3} x_{6} x_{8}, x_{1} x_{5} x_{9}, x_{2} x_{8} x_{9}\right) \subseteq$ $\mathbf{k}\left[x_{1}, \ldots, x_{9}\right]$. We consider the Betti diagrams of the resolutions of the first few powers $I^{d}$ of $I$. The diagrams have been shifted to only show nonzero Betti numbers in the resolution of $I^{d}$.

$\begin{array}{ccccccc}I & & & & & & \\ & - & 1 & 2 & 3 & 4 & 5 \\ \text { total: } & 5 & 10 & 9 & 3 & . \\ 2: & 5 & . & . & . & . \\ 3: & . & 6 & . & . & . \\ 4: & . & 4 & 9 & 3 & .\end{array}$

$\begin{array}{ccccccc}I^{2} & & & & & & \\ & - & 1 & 2 & 3 & 4 & 5 \\ & \text { total } & 15 & 41 & 39 & 12 & . \\ 5: & 15 & . & . & . & . \\ 6: & . & 33 & 12 & . & . \\ & & . \\ 7: & . & 8 & 27 & 12 & .\end{array}$


$I^{3}$

\begin{tabular}{|c|c|c|c|c|c|}
\hline- & 1 & 2 & 3 & 4 & 5 \\
\hline total: & 35 & 117 & 121 & 39 & 1 \\
\hline 8: & 35 & . & . & . & . \\
\hline 9: & . & 105 & 67 & 9 & . \\
\hline 10: & . & 12 & 54 & 30 & 1 \\
\hline & & & & & \\
\hline- & 1 & 2 & 3 & 4 & 5 \\
\hline total: & 126 & 545 & 645 & 240 & 15 \\
\hline 14: & 126 & $\cdot$ & . & . & $\cdot$ \\
\hline 15: & . & 525 & 510 & 135 & 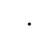 \\
\hline 16: & . & 20 & 135 & 105 & 15 \\
\hline
\end{tabular}

$I^{4}$

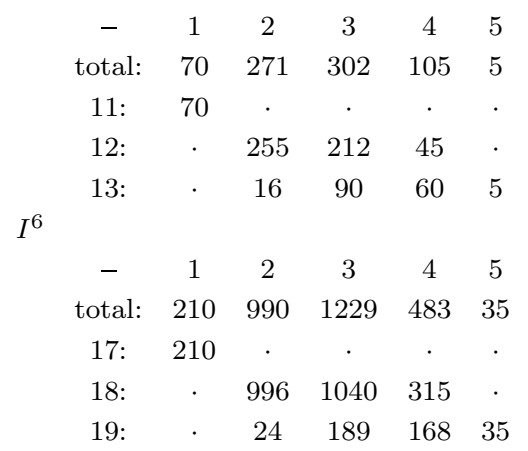

We can see the stabilized shape of the powers of $I^{d}$ will be:

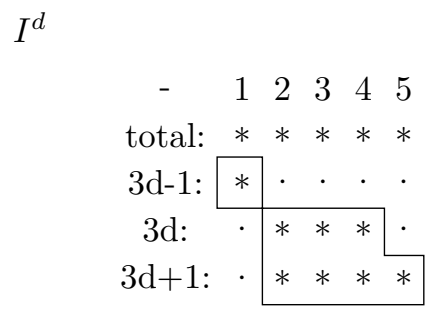

Unfortunately, Theorem 4.1 does not guarantee that powers of our ideals $I^{d}$ will have linear resolutions if the resolution of $I^{l}$ is linear for some $l$ with $d>l$. As a counterexample, we have the following example (due to Sturmfels):

Example 1.4 [7]. Set $I=($ def, cef, cdf, cde, bef, bcd,acf,ade $) \subseteq$ $\mathbf{k}[a, b, c, d, e, f]$. The ideal $I$ has linear resolution and linear quotients with respect to the ordering given above, but $I^{2}$ fails to be linear. We include the Betti tables of $I$ and $I^{2}$ here. 
$I$

$\begin{array}{ccccc}- & 0 & 1 & 2 & 3 \\ \text { total: } & 1 & 8 & 11 & 4 \\ 0: & 1 & . & . & . \\ 1: & . & . & . & . \\ 2: & . & 8 & 11 & 4\end{array}$

$I^{2}$

$\begin{array}{cccccccc}- & 0 & 1 & 2 & 3 & 4 & 5 & 6 \\ \text { total: } & 1 & 36 & 85 & 79 & 38 & 10 & 1 \\ 0: & 1 & . & . & . & . & . & . \\ 1: & . & . & . & . & . & . & . \\ 2: & . & . & . & . & . & . & . \\ 3: & . & . & . & . & . & . & . \\ 4: & . & . & . & . & . & . & . \\ 5: & . & 36 & 84 & 75 & 32 & 6 & . \\ 6: & . & . & 1 & 4 & 6 & 4 & 1\end{array}$

More generally, Conca provided a class of ideals $I_{k}$ which have linear quotients (and hence, linear resolutions) until the $k$ th power, then have nonlinear resolutions for all powers higher than $k[2]$. This implies that, for an ideal $I$, the shapes of Betti tables of $I, I^{2}, \ldots, I^{d}$ and $I^{d+1}$ need not satisfy any chain of inclusions, though they eventually stabilize for some $I^{D}$.

We also provide an upper bound for the Betti numbers of powers of an equigenerated ideal $I$ in terms of the Betti numbers of the Rees ideal of $I$ as follows.

Theorem 1.5 (Theorem 3.1). Let $I=\left(f_{0}, f_{1}, \ldots, f_{k}\right) \subseteq R=$ $\mathbf{k}\left[x_{1}, \ldots, x_{N}\right]$ with $f_{i}$ homogeneous of degree $r$. Let $\mathcal{R}(I)$ be the Rees algebra of $I$ over the ring $S=\mathbf{k}\left[x_{1}, \ldots, x_{N}, w_{0}, \ldots, w_{k}\right]$ with bigrading $\operatorname{deg}\left(x_{i}\right)=(1,0)$ and $\operatorname{deg}\left(w_{i}\right)=(0,1)$. Then

$$
\beta_{i, j+r d}\left(I^{d}\right) \leq \sum_{m=0}^{d}\left(\begin{array}{c}
d+k-m \\
d-m
\end{array}\right) \beta_{i,(j, m)}(\mathcal{R}(I))
$$

holds for all $i, j, d$.

The proof follows from a careful examination of the restriction of a minimal resolution of $\mathcal{R}(I)$ to bidegrees $(*, d)$. We give a name to the smallest $D$ for which this stabilization occurs:

Definition 1.6. Let $I$ be a homogeneous equigenerated ideal in a polynomial ring $R$. Let the stabilization index Stab $(I)$ of $I$ be the 
smallest such $D$ such that, for all $d \geq D$,

$$
\beta_{i, j+r d}\left(I^{d}\right) \neq 0 \Longleftrightarrow \beta_{i, j+r D}\left(I^{D}\right) \neq 0 .
$$

Finding Stab $(I)$ directly in terms algebraic properties of $I$ remains open. Areas of future research include producing explicit Stab $(I)$ for other classes of ideals or providing sharper bounds for Stab $(I)$ than those offered here.

After the initial submission of this work, the author has been informed that one of Herzog's students, Pooja Singla, obtained in her thesis a very similar result.

\section{Rees algebras of equigenerated ideals.}

2.1. Rees algebras and degree restrictions. One common technique used in investigating powers $I^{n}$ of an ideal $I$ involves passing to the Rees algebra of $I$. The Rees algebra $\mathcal{R}(I)$ of an ideal $I$ is an object which captures the ideal $I$ and all of its powers.

Definition 2.1. Let $I=\left(f_{0}, f_{1}, \ldots, f_{k}\right) \subseteq R=\mathbf{k}\left[x_{1}, \ldots, x_{N}\right]$. The Rees algebra $\mathcal{R}(I)$ of $I$ is

$$
\mathcal{R}(I)=R \oplus I t \oplus I^{2} t^{2} \oplus I^{3} t^{3} \oplus \cdots \oplus I^{n} t^{n} \oplus \cdots .
$$

This is occasionally denoted $R[I t]$.

In general, we will use a presentation of $\mathcal{R}(I)$ as a quotient module of the ring $S=R\left[w_{0}, w_{1}, \ldots, w_{k}\right]=\mathbf{k}\left[x_{1}, \ldots, x_{N}, w_{0}, w_{1}, \ldots, w_{k}\right]$.

Proposition $2.2[9]$. Let $I=\left(f_{1}, \ldots, f_{k}\right) \subseteq R=\mathbf{k}\left[x_{1}, \ldots, x_{N}\right]$, and let $\mathcal{R}(I)$ be its Rees algebra. Then $\mathcal{R}(I)=R\left[w_{1}, \ldots, w_{k}\right] / L=$ $\mathbf{k}\left[x_{1}, \ldots, x_{N}, w_{0}, w_{1}, \ldots, w_{k}\right] / L$, with presentation ideal

$$
L=\left(f_{i}-w_{i} t: 1 \leq i \leq k\right) S[t] \cap S .
$$

If $S=\mathbf{k}\left[x_{1}, \ldots, x_{N}, w_{1}, \ldots, w_{k}\right]$, and $\mathcal{R}(I)=S / L$, then $L$ is the Rees ideal of $I$. 
2.2. Resolutions and bigradings of Rees algebras. Taking a resolution (with an appropriately chosen bigrading) of $L$ gives resolutions of all powers of $L$ and can be used to bound or explicitly compute Betti numbers $\beta_{i, j}\left(I^{n}\right)$ for all $n$.

We will assume throughout this paper that $I=\left(f_{0}, f_{1}, \ldots, f_{k}\right)$ is an equigenerated ideal of degree $r$ in $R=\mathbf{k}\left[x_{1}, \ldots, x_{N}\right]$. Notationally, we set $\mathcal{R}(I)=S / L$ with $L$ the Rees ideal of $I$ and $S=\mathbf{k}\left[x_{1}, \ldots, x_{N}, w_{0}, w_{1}, \ldots, w_{k}\right]$.

We bigrade $\mathcal{R}(I)$ by $\operatorname{deg}\left(x_{i}\right)=(1,0)$ and $\operatorname{deg}\left(w_{i}\right)=(0,1)$ and take the minimal graded free resolution of $\mathcal{R}(I)$ with respect to this grading.

$$
\begin{aligned}
\mathcal{F}: \mathcal{R}(I) \leftarrow S \leftarrow \bigoplus_{(j, m)} S(-j,-m)^{\beta_{1,(j, m)}} & \leftarrow \cdots \\
& \leftarrow \bigoplus_{(j, m)} S(-j,-m)^{\beta_{p,(j, m)}} \leftarrow 0 .
\end{aligned}
$$

Restricting to the strand $(*, d)$, we obtain a (possibly nonminimal) resolution of $I^{d}$ :

$$
\begin{aligned}
\mathcal{F}_{\lceil}: I^{d} \leftarrow S_{(*, d)} \leftarrow \bigoplus_{(j, m)} S(-j,-m)_{(*, d)}^{\beta_{1,(j, m)}} \leftarrow \cdots \\
\\
\leftarrow \bigoplus_{(j, m)} S(-j, m)_{(*, d)}^{\beta_{p,(j, m)}} \leftarrow 0 .
\end{aligned}
$$

Tensoring this resolution with $\mathbf{k}$ and taking the homology of the maps gives us $\operatorname{dim} \operatorname{Tor}_{i}^{R}\left(\mathbf{k}, I^{d}\right)_{j+r d}=\beta_{i, j+r d}\left(I^{d}\right)$. This shift in the indices of $\beta_{i, j+r d}\left(I^{d}\right)$ accounts for the shift in grading to agree with that of $R$ while viewing $I^{d}$ as an $R$ module.

Alternately, we could have first tensored with $S / M$ for $M=$ $\left(x_{1}, \ldots, x_{N}\right)$, taken homology of our maps, then restricted in degrees. This will give us modules $\operatorname{Tor}_{i}^{S}(S / M, \mathcal{R}(I))_{j}$, and as these two actions commute, we have that

$$
\begin{aligned}
\operatorname{Tor}_{i}^{S}(S / M, \mathcal{R}(I))_{(j, d)} & =\operatorname{Tor}_{i}^{R}\left(S / M, I^{d}\right)_{j+r d} \\
& =\operatorname{Tor}_{i}^{R}\left(\mathbf{k}, I^{d}\right)_{j+r d}
\end{aligned}
$$

where the second equality follows from $S / M \cong \mathbf{k}$ as an $R$-module. 
Hence, we have that all Betti numbers of higher powers can be written in terms of the dimensions of the bigraded modules $\operatorname{Tor}_{i}^{S}(S / M, \mathcal{R}(I))$, as

$$
\beta_{i, j+r d}\left(I^{d}\right)=\operatorname{dim} \operatorname{Tor}_{i}^{S}(S / M, \mathcal{R}(I))_{(j, d)} .
$$

3. Bounds on Betti numbers of powers of ideals. We resolve the Rees algebra $\mathcal{R}(I)$ and restrict to fixed $w$-degree strands to produce explicit bounds on the Betti numbers of $I^{d}$.

Theorem 3.1. Let $I=\left(f_{0}, f_{1}, \ldots, f_{k}\right) \subseteq R=\mathbf{k}\left[x_{1}, \ldots, x_{N}\right]$ with all $f_{i}$ homogeneous of degree $r$. Let $\mathcal{R}(I)$ be the Rees algebra of $I$ in ring $S=\mathbf{k}\left[x_{1}, \ldots, x_{N}, w_{0}, \ldots, w_{k}\right]$ with bigrading $\operatorname{deg}\left(x_{i}\right)=(1,0)$ and $\operatorname{deg}\left(w_{i}\right)=(0,1)$. Then

$$
\beta_{i, j+d r}\left(I^{d}\right) \leq \sum_{m=0}^{d}\left(\begin{array}{c}
d+k-m \\
d-m
\end{array}\right) \beta_{i,(j, m)}(\mathcal{R}(I))
$$

holds for all $i, j, d$.

Proof. We take a minimal free resolution of $\mathcal{R}(I)$ and consider the degree restricted strand used in Section 2:

$$
\begin{aligned}
\mathcal{F}_{\lceil}: I^{d} \leftarrow S_{(*, d)} \leftarrow \bigoplus_{(j, m)} S(-j,-m)_{(*, d)}^{\beta_{1,(j, m)}} \leftarrow \cdots \\
\leftarrow \bigoplus_{(j, m)} S(-j,-m)_{(*, d)}^{\beta_{p,(j, m)}} \leftarrow 0 .
\end{aligned}
$$

Let $T=\mathbf{k}\left[w_{0}, w_{1}, \ldots, w_{k}\right]$ be the polynomial ring in the $w_{i}$-variables. Then we can rewrite our bigraded pieces $S(-j,-m)=R(-j) \otimes T(-m)$. Then, in a fixed strand $(*, d)$, we have:

$$
\begin{aligned}
\mathcal{F}_{\lceil}: I^{d} \leftarrow R \otimes T_{d} \leftarrow \bigoplus_{(j, m)} R(-j) \otimes T(-m)_{d}^{\beta_{1,(j, d)}} \leftarrow \cdots \\
\leftarrow \bigoplus_{(j, m)} R(-j) \otimes T(-m)_{d}^{\beta_{p,(j, d)}} \leftarrow 0 .
\end{aligned}
$$


It remains to count the dimension over $R$ of the $i$ th module

$$
F_{i}=\bigoplus_{(j, m)} R(-j) \otimes T(-m)_{d}^{\beta_{i,(j, m)}(\mathcal{R}(I))}
$$

in a fixed degree $j+r d$ of the resolution. Finally, the dimension of $T(-m)_{d}$ is the number of degree $d-m$ monomials in a polynomial ring in $k+1$ variables, or

$$
\left(\begin{array}{c}
d+k-m \\
k
\end{array}\right)
$$

So we have that

$$
\beta_{i, j+r d}\left(I^{d}\right) \leq \sum_{m=0}^{d}\left(\begin{array}{c}
d+k-m \\
k
\end{array}\right) \beta_{i,(j, m)}(\mathcal{R}(I))
$$

proving the theorem.

We see from this that the Betti diagram of $I^{d}$ sits inside an (appropriately degree shifted) table coming from the Betti diagram of the resolution of $\mathcal{R}(I)$. This implies that the number of nonzero graded Betti numbers of $I^{d}$ is bounded independent of the power $d$. We refine this rough bound in the following section.

4. Betti diagrams of powers of equigenerated ideals. We are now ready to prove the main theorem:

Theorem 4.1 (Betti tables of powers of equigenerated ideals). Let $I=\left(f_{0}, f_{1}, \ldots, f_{k}\right) \subseteq \mathbf{k}\left[x_{1}, \ldots, x_{N}\right]=R$ be an equigenerated ideal of degree $r$. Then there exists a $D$ such that, for all $d>D$, we have

$$
\beta_{i, j+r d}\left(I^{d}\right) \neq 0 \Longleftrightarrow \beta_{i, j+r D}\left(I^{D}\right) \neq 0
$$

Proof. From the calculation in Section 2, we have that

$$
\beta_{i, j+r d}\left(I^{d}\right)=\operatorname{dim} \operatorname{Tor}_{i}^{S}(S / M, \mathcal{R}(I))_{(j, d)}
$$

The $\operatorname{Tor}_{i}(S / M, \mathcal{R}(I))$ are finitely generated bigraded $S$-modules. We decompose them into bigraded components in the following way.

Let $M_{i}:=\operatorname{Tor}_{i}(S / M, \mathcal{R}(I))$ and $M_{i j}:=\left(M_{i}\right)_{(j, *)}$. 
Lemma 4.2. The $M_{i j}$ are finitely generated graded $T$-modules, where

$$
T=\mathbf{k}\left[w_{0}, w_{1}, \ldots, w_{k}\right]
$$

is the polynomial ring in the $w_{i}$-variables.

Proof. As $S$ is a Noetherian ring, we have that $S / M$ and $\mathcal{R}(I)$ are Noetherian $S$-modules. From this, we have that $\operatorname{Tor}_{i}^{S}(S / M, \mathcal{R}(I))$ is a finitely generated $S / M$-module. We also note that, in a fixed strand degree strand $(j, *)$, we have

$$
(S / M)_{(j, *)} \cong(R / M)_{j} \otimes T_{*} \cong \mathbf{k} \otimes T_{*} \cong T,
$$

so in a fixed $x$-degree strand $(j, *)$, we have that $\operatorname{Tor}_{i}^{S}(S / M, \mathcal{R}(I))_{(j, *)}$ is finitely generated as a $T$-module.

As a $T$-module, each $M_{i j}$ has a Hilbert polynomial

$$
P_{i j}(d):=P_{M_{i j}}(d)=\operatorname{dim}\left(M_{i}\right)_{(j, d)}
$$

for all $d \geq d_{i j}$, with $d_{i j}$ the regularity of $M_{i j}$. Hence, for all $d \geq d_{i j}$, $P_{M_{i j}}$ is either identically zero or not.

Note that $D=\max _{i, j}\left\{d_{i j}\right\}$ will be an upper bound for $\operatorname{Stab}(I)$, provided such a maximum exists.

Lemma 4.3. There are only finitely many nonzero $M_{i j}$.

Proof. That only finitely many $M_{i}$ are nonzero follows from

$$
\beta_{i, j+r d}\left(I^{d}\right)=\operatorname{dim} \operatorname{Tor}_{i}^{S}(S / M, \mathcal{R}(I))_{(j, d)} .
$$

As the projective dimension of all powers $I^{d}$ is bounded by $N$, the number of variables in our original ring, $\operatorname{Tor}_{i}^{S}(S / M, \mathcal{R}(I))=0$ for all $i>N$.

We now consider a fixed $M_{i}$. Theorem 3.1 gave a bound on the Betti numbers of $I^{d}$ depending on the Betti numbers of $\mathcal{R}(I)$,

$$
\beta_{i, j+r d}\left(I^{d}\right) \leq \sum_{m=0}^{d}\left(\begin{array}{c}
d+k-m \\
d-m
\end{array}\right) \beta_{i,(j, m)}(\mathcal{R}(I))
$$


As for a fixed $i$, the number of nonzero Betti numbers of $\mathcal{R}(I)$ must be finite, there can be only finitely many $j$ such that $\beta_{i,(j, m)}(\mathcal{R}(I)) \neq 0$. This implies that, for $j$ outside of this set, $\beta_{i, j+r d}\left(I^{d}\right) \leq \sum_{m=0}^{d} 0$ for all $d$, which implies $\beta_{i, j+r d}\left(I^{d}\right)=0$. So $M_{i j}=0$ except for a finite number of cases.

This completes the proof of the lemma.

By Lemma 4.3, we have that the maximum

$$
D=\max _{i, j}\left\{d_{i j}\right\}
$$

exists. Hence, we have that

$$
\operatorname{dim} \operatorname{Tor}_{i}(S / M, \mathcal{R}(I))_{(*, d)}=P_{M_{i}}(d)
$$

is a polynomial function for all $d>D$. We note that, for all $d>D$,

$$
\beta_{i, j+d r}\left(I^{d}\right)=\operatorname{dim}\left(M_{i}\right)_{(j, d)}=P_{M_{i, j}}(d)>0
$$

if and only if

$$
\beta_{i, j+D r}\left(I^{D}\right)=\operatorname{dim}\left(M_{i}\right)_{(j, D)}=P_{M_{i, j}}(D)>0,
$$

completing the proof.

The techniques used throughout the proof of Theorem 4.1 were similar to those seen in $[\mathbf{1}, \mathbf{3}, \mathbf{8}]$, but extend their results to a classification of all possible nonzero graded Betti numbers of powers of an equigenerated ideal $I$.

5. Stabilization index of $I$. The bound $D$ produced in Theorem 4.1 is not sharp, and finding the smallest such $D$, which we will call the stabilization index of $I$, Stab $(I)$, in terms of combinatorial data of $I$ is a subject of future research.

Definition 5.1. Let $I$ be a homogeneous ideal equigenerated in degree $r$ in a polynomial ring $R$. Let $\operatorname{Stab}(I)$ be the smallest such $D$ such that for all $d \geq D$,

$$
\beta_{i, j+r d}\left(I^{d}\right) \neq 0 \Longleftrightarrow \beta_{i, j+r D}\left(I^{D}\right) \neq 0 .
$$


Finding a sharp formula for $\operatorname{Stab}(I)$ for ideals $I$ remains open, even in the case of square-free monomial ideals generated in degree 2 (the so-called edge ideals) with a linear resolution.

One reason that this class initially appears a good candidate for calculation of Stab $(I)$ is due to a result of Herzog, Hibi and Zheng [5] which states the following:

Theorem 5.2 [5]. Let $I$ be a quadratic monomial ideal of the polynomial ring. The following are equivalent:

(1) I has a linear resolution,

(2) I has linear quotients,

(3) $I^{k}$ has a linear resolution for all $k \geq 1$.

So for ideals generated in degree 2 with a linear resolution, it is known that the regularity of such ideals stabilizes at the first power, i.e., $\operatorname{reg}\left(I_{G}^{d}\right)=2 d$ for all $I_{G}$ with $\operatorname{reg}\left(I_{G}\right)=2$. This stabilization of regularity does not guarantee a stabilization of the Betti tables, even in this instance.

Example 5.3. Consider the graph $G$ of a 4 -cycle with a single attached leaf,

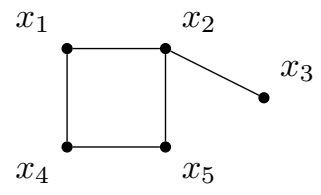

given by ideal

$$
I=\left(x_{1} x_{2}, x_{1} x_{4}, x_{2} x_{3}, x_{2} x_{5}, x_{4} x_{5}\right) \subseteq \mathbf{k}\left[x_{1}, \ldots, x_{5}\right] .
$$

This has a linear resolution (and hence, all powers have linear resolutions), but $\operatorname{Stab}\left(I_{G}\right)>1$ : 
I

$$
\begin{array}{ccccc}
- & 0 & 1 & 2 & 3 \\
\text { total: } & 1 & 5 & 6 & 2 \\
0: & 1 & \cdot & \cdot & \cdot \\
1: & \cdot & 5 & 6 & 2
\end{array}
$$

$$
\begin{array}{ccccccc}
I^{2} & & & & & & \\
& - & 0 & 1 & 2 & 3 & 4 \\
& \text { total: } & 1 & 14 & 24 & 13 & 2 \\
0: & 1 & . & . & . & . \\
1: & . & . & . & . & . \\
2: & . & . & . & . & . \\
2: & . & 14 & 24 & 13 & 2
\end{array}
$$

Through examination of subsequent powers, we note that $\operatorname{Stab}(I)=2$.

This indicates that known formulas bounding (or explicitly providing) regularity of powers of ideals are unlikely to provide bounds on Stab $(I)$.

5.1. Areas of future research. We would like to answer the following questions in subsequent work on these stabilization indices:

(1) Do formulas for Stab $(I)$ exist for squarefree monomial ideals? Do they relate to the dimensions of the facet complex or the StanleyReisner complex?

(2) For such squarefree monomial ideals, does $\operatorname{Stab}\left(I_{\Delta}\right)$ have a topological interpretation in terms of $\Delta_{\mathrm{pol}\left(I^{n}\right)}$, the Stanley-Reisner complex of the polarization of $I^{n}$ ?

(3) Does there exist a class of ideals for which the $D$ produced in Theorem 4.1 is the sharp bound, i.e., $D=\operatorname{Stab}(I)$ ?

Aside from the stabilization index, the shapes of chain of Betti tables leading up to the stabilized Betti table appear fairly interesting. Generally, the shapes of Betti tables of powers of homogeneous equigenerated ideals seem to be unimodal, in the following sense:

Conjecture 5.4. Let $I \subseteq R$ be a homogeneous ideal generated in degree $r$. Then for each pair of indices $(i, j)$ there exist $1 \leq D_{1} \leq D_{2} \leq$ $\infty$ such that, for all $d$ with $D_{1} \leq d \leq D_{2}$,

$$
\beta_{i, j+d r}\left(I^{d}\right) \neq 0
$$

and for all $d<D_{1}$ or $D_{2}<d$,

$$
\beta_{i, j+d r}\left(I^{d}\right)=0
$$


Note that this conjecture does not state that there exists a pair of powers $D_{1}, D_{2}$ such that for all pairs $(i, j), \beta_{i, j+d r}$ are nonzero for $D_{1} \leq d \leq D_{2}$ and zero outside of this range.

The behavior which motivated the conjecture was observing, for every example the author has encountered to date, the following: For an ideal $I$, let

$$
b_{d}=\beta_{i, j+d r}\left(I^{d}\right) .
$$

The Betti number sequence of powers $I^{d}$ for each pair of indices $(i, j)$,

$$
\left\{b_{1}, b_{2}, b_{3}, \ldots, b_{d-1}, b_{d}, b_{d+1}, \ldots\right\}
$$

has been of the form

$$
\left\{0,0, \ldots, 0, b_{d}, b_{d+1}, \cdots\right\}
$$

where all $b_{i} \neq 0$ for $d \leq i$, or of the form

$$
\left\{b_{0}, b_{1}, \ldots, b_{d-1}, 0,0, \cdots\right\}
$$

where $b_{i} \neq 0$ for all $0 \leq i \leq d-1$.

This amounts to the $T$-modules $M_{i j}=\operatorname{Tor}_{i}^{S}(S / M, \mathcal{R}(I))_{(j, *)}$ defined above never vanishing in some degree $d$ with another generator of $M_{i j}$ occurring in a higher degree $d+s$.

To date, several hundred thousand randomly generated monomial, binomial and other ideals have been run through Macaulay 2 without a counterexample arising. Code to produce random ideals of various forms and examine these Betti sequences is available on the author's website.

Making progress on this conjecture (or providing a counterexample with pathological Betti diagram behavior) would require a much tighter understanding of the $M_{i j}=\operatorname{Tor}_{i}^{S}(S / M, \mathcal{R}(I))_{(j, *)}$. These $M_{i j}$ seem to carry interesting structure, and investigating the connections between $M_{i j}$ and the geometry of the ideal $I$ and its Rees algebra $\mathcal{R}(I)$ is another area of future interest.

Acknowledgments. The author would like to thank her advisor, Mike Stillman, for his many helpful conversations and comments throughout the course of this work. This paper would not have been 
possible without his many suggestions. The author would also like to thank Greg Muller for discussions on some of the most delicate points of the homological algebra involved.

\section{REFERENCES}

1. Keivan Borna, On linear resolution of powers of an ideal, Osaka J. Math. 46 (2009), 1047-1058.

2. Aldo Conca, Regularity jumps for powers of ideals, Commutative algebra, Lect. Notes Pure Appl. Math. 244, Chapman \& Hall/CRC, Boca Raton, FL, 2006.

3. S. Dale Cutkosky, Jürgen Herzog and Ngô Viêt Trung, Asymptotic behaviour of the Castelnuovo-Mumford regularity, Comp. Math. 118 (1999), 243-261.

4. Ralf Fröberg, On Stanley-Reisner rings, in Topics in algebra, Part 2 (Warsaw, 1988), Banach Center Publ. 26, PWN,Warsaw, 1990.

5. J. Herzog, T. Hibi and X. Zheng, Monomial ideals whose powers have a linear resolution, ArXiv Math. e-prints (2003).

6. Tim Römer, Homological properties of bigraded algebras, Ill. J. Math. 45 (2001), 1361-1376.

7. B Sturmfels, Four counterexamples in combinatorial algebraic geometry, J. Algebra 230 (2000), 282-294.

8. Irena Swanson, Powers of ideals. Primary decompositions, Artin-Rees lemma and regularity, Math. Ann. 307 (1997), 299-313..

9. Wolmer V. Vasconcelos, Arithmetic of blowup algebras, Lond. Math. Soc. Lect. Note Ser. 195, Cambridge University Press, Cambridge, 1994.

Department of Mathematics, Hood College, Frederick, MD 21701

Email address: whieldon@hood.edu 